\title{
TOTAL AND INCREMENTAL ITERATION FORCE RECOVERY PROCEDURE FOR THE NONLINEAR ANALYSIS OF FRAMED STRUCTURES
}

\author{
Fawu Wang ${ }^{1, *}$ and Yaopeng Liu ${ }^{2}$ \\ ${ }^{l}$ Department of Civil Engineering, Nanjing University of Aeronautics and Astronautics \\ Yudao Street, Nanjing, China \\ ${ }^{2}$ Department of Civil and Structural Engineering, The Hong Kong Polytechnic University \\ Hunghom, Kowloon, Hong Kong \\ *(Corresponding author: E-mail: fwwang@nuaa.edu.cn)
}

Received: 22 May 2008; Revised: 6 August 2008; Accepted: 20 August 2008

\begin{abstract}
Co-rotational formulation is suitable for the incremental-iterative nonlinear analysis of framed structure which can be divided into three stages: the predictor, corrector and error-checking phases. The corrector or force recovery procedure determines the accuracy of the solution while the predictor affects only the convergence speed and direction of iteration. This paper details the formulation of the incremental-iterative nonlinear analysis of framed structure with PEP element and reviews the present existing force recovery procedures. A new force recovery method is proposed and compared with total secant iteration method and pure incremental method. It is found through three numerical examples that this incremental iteration force recovery procedure has a convergence rate comparable to total secant iteration method and it is recommended for path-dependent analysis.
\end{abstract}

Keywords: Nonlinear analysis; framed structures; force recovery procedure; steel frame

\section{INTRODUCTION}

The nonlinear analysis of 3Dimensional framed structures has been extensively studied by various researchers. Geometric nonlinearity is an important nonlinear source because of the slenderness of most framed structure members. Plasticity and semi-rigid connections also play an important role for nonlinear analysis.. To obtain the nonlinear response of framed structure accurately, the incremental-iterative technique provides a unique solution under a prescribed load level. In practice the incremental and iterative solution strategy are employed to trace the load vs. deflection path of a structure.

The incremental-iterative nonlinear analysis of framed structure can be divided into three stages: the predictor, corrector and error-checking phases. The task of the predictor stage is to estimate the structural displacements under a given load increment. The corrector is concerned with recovery of the element forces for the obtaining element displacements and deformations. In the error-checking phase the unbalanced forces are computed by comparing the internal resistant forces with the applied loads. The predictor affects only the convergence and direction of iteration, while the corrector determines the accuracy of solution [1].

In this paper the recovery procedure of the element forces is investigated and three force recovery methods, namely as the total secant iteration, pure incremental and incremental secant iteration force recovery procedures are described. The advantages and limitations of these three force recovery methods are compared. 


\section{CO-ROTATIONAL FORMULATION}

It is well known that three Lagrangian kinematic descriptions are available for the finite element analysis of geometrically nonlinear structures. Namely they are the total Lagrangian, the updated Lagrangian, and the co-rotational formulation. The reference configuration in the total Lagrangian formulation is the initial un-deformed configuration $C_{0}$, whereas in updated Lagrangian formulation is the last known deformed configuration $C_{l}$. In co-rotational formulation the motion is split into two components: rigid body motion and deformational motion. The rigid body motion is represented by a reference frame which is rigidly attached to the end of the element and continuously rotates with the element as shown in Figure 1. Consequently an important advantage of the co-rotational formulation is that the volume integrations are generally performed over the simple un-deformed shape of the element. This reference frame can be viewed as an intermediary co-rotated configuration $C^{R}$ for clear physical meaning although from a mathematical standpoint the explicit presence of this co-rotated configuration between initial and deformed configuration is unnecessary. The motion can be measured between the deformed configuration and the co-rotated configuration $C^{R}$. The essence of the co-rotational formulation is that the deformational motion is relatively small so that simplified strain measurement such as engineering strain can be used to formulate the strain energy of the element. When this deformational motion is large such that more complicated strain measurements are needed, the co-rotational formulation has little advantage over the total Lagrangian formulation or the updated Lagrangian formulation.

The co-rotational formulation is especially useful in the formation for geometrically nonlinear analysis of frame structures as the reference frame of every element can be easily determined by the positions of two nodes of the element. The displacement of node $\mathbf{a}(\mathbf{a}=1, \ldots, \mathrm{N})$ of the structures can be represented by a set $\mathbf{v}_{\mathrm{a}}=\mathrm{s}$. In contract to the translational displacement $\mathbf{d}_{\mathrm{a}}$ which can be represented by a vector and is always additive, the rotational displacement $\mathbf{R}_{\mathrm{a}}$ does not transform as a vector when finite rotations are considered. The key operation of the co-rotational formulation is to extract the deformational components of the translations and rotations for each node when the incremental nodal displacement $\Delta \mathbf{v}_{\mathrm{a}}$ is given by the predictor. The deformation extraction operation also involves the updating of element reference frame and nodal rotations. Several methods are available to deal with large rotations in different situations as listed in the next section.

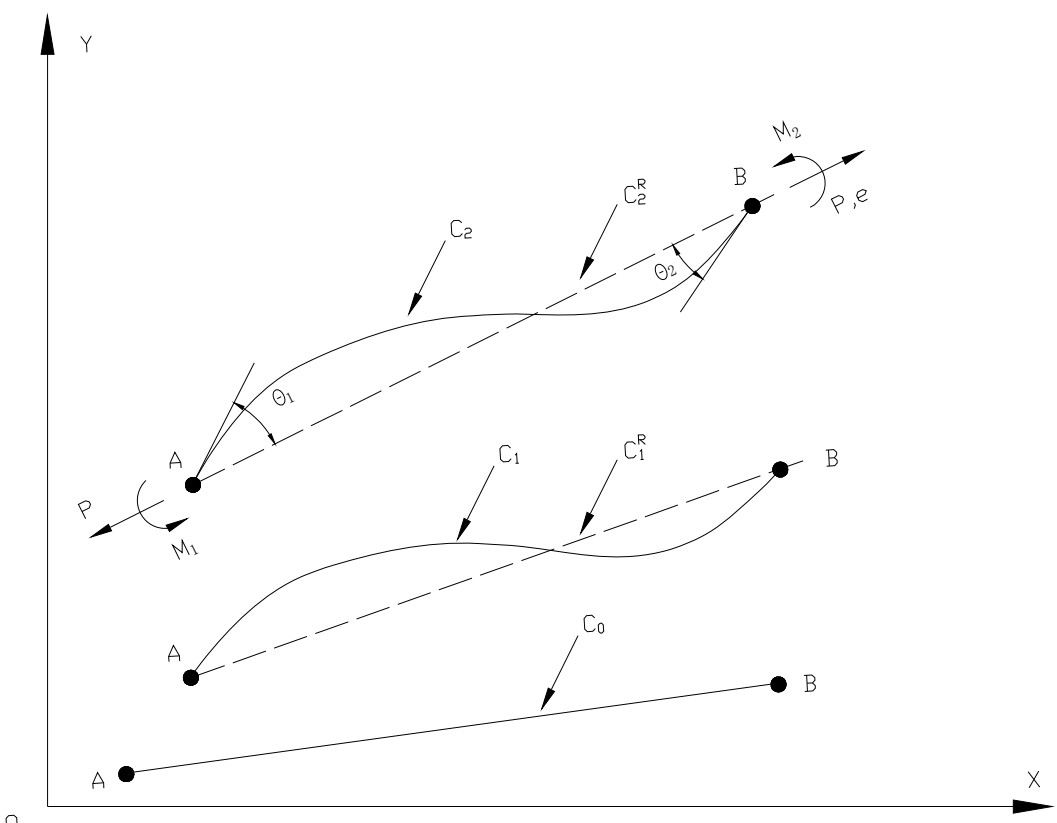

Figure 1. Co-rotational Formulation 


\section{LARGE ROTATION CONSIDERATION}

Chan [2] compared three different methods considering large rotations and found that the joint orientation approach is a reliable formulation for genuinely large rotations in three-dimensional space while simplified methods can be used for small or moderately large rotations, which is the case of most practical engineering framed structures.

\subsection{Large Rotations, Small but Finite Deformational Rotations}

Strictly speaking, unit co-rotational triads which represents the directional matrix should be attached to each node and element to account for finite or large rotations. The nodal rotations $\mathbf{R}_{\mathrm{a}}$ can be presented by the position of unit triads and should be updated through the incremental rotations with Rodrigues Formula. To this, several forms of rotation transformation matrix can be used. The deformational rotations can be determined by the angle of the rotation of nodal triads relative to the rigid rotation of the element. The rigid body rotation can be represented by the rotation of the element reference frame described by a set of Cartesian coordinate system where the $x$ axis is chosen to pass through two end nodes of the element and $y$ and $z$ axes are chosen to be the principal directions of the cross section at the initial un-deformed state. This method was firstly used by Oran [3] and has been detailed by Izzuddin and Elnashai [4] and others. The mathematical deduction can refer to the paper by Felippa and Haugen [5].

\subsection{Moderately Large Rotations, Small Deformational Rotations}

With moderately large rotations but small deformational rotations, which is the case of most engineering structures in practice, the above method is applicable but a more concise method can be used. Instead of joint orientation triads, only the element orientation triad is used in element orientation method and the incremental local deformational displacements can be obtained through the incremental total displacements with incremental rigid body rotations eliminated. The total deformational rotations can be accumulated by every increment and so do the rigid body rotations. This approach has been used by many researchers [6-8] for its simplicity and easy implementation.

The rigid body rotations increments about local $y$ and $z$ axis can be obtained through the nodal incremental displacements $\mathbf{v}_{l}$ in local element coordinate system as,

$$
\begin{aligned}
& \sin \Delta \Theta_{z}=\left(\Delta v_{2}-\Delta v_{1}\right) / L \\
& \sin \Delta \Theta_{y}=-\left(\Delta w_{2}-\Delta w_{1}\right) / L \\
& \Delta \Theta_{x}=\left(\Delta \alpha_{2}+\Delta \alpha_{1}\right) / 2
\end{aligned}
$$

Where $L$ is the element chord length by

$$
L=\sqrt{\left(X_{E}\right)^{2}+\left(Y_{E}\right)^{2}+\left(Z_{E}\right)^{2}}
$$

Where $X_{\mathrm{E}}, Y_{\mathrm{E}}, Z_{\mathrm{E}}$ and $X_{\mathrm{E}}^{0}, Y_{\mathrm{E}}^{0}, Z_{\mathrm{E}}^{0}$ is the global coordinate difference between two nodes of the element in current and initial configuration and

$$
X_{E}=X_{E}^{0}+\Delta u_{2}-\Delta u_{1}
$$




$$
\begin{aligned}
& Y_{E}=Y_{E}^{0}+\Delta v_{2}-\Delta v_{1} \\
& Z_{E}=Z_{E}^{0}+\Delta w_{2}-\Delta w_{1}
\end{aligned}
$$

The incremental element nodal displacements $\Delta \mathbf{v}_{g}$ in Equation below can be obtained by a standard finite element procedure from the incremental structural nodal displacements $\Delta \mathbf{v}_{\mathrm{a}}$ which is determined by an incremental-iterative predicting process. :

$$
\Delta \mathbf{v}_{g}=\left\{\Delta u_{1}, \Delta v_{1}, \Delta w_{1}, \Delta \alpha_{1}, \Delta \beta_{1}, \Delta \gamma_{1}, \Delta u_{2}, \Delta v_{2}, \Delta w_{2}, \Delta \alpha_{2}, \Delta \beta_{2}, \Delta \gamma_{2}\right\}^{T}
$$

Then the local incremental deformation $\Delta \mathbf{u}_{l}$ can be obtained by

$$
\begin{aligned}
& \Delta \mathrm{u}_{l}=\left\{\Delta \theta_{1 y}, \Delta \theta_{1 z}, \Delta \theta_{1 z}, \Delta \theta_{1 z}, \Delta e, \Delta \theta_{x}\right\}^{T} \\
& \Delta \theta_{1 y}=\Delta \beta_{1}-\Delta \Theta_{y} \\
& \Delta \theta_{1 z}=\Delta \gamma_{1}-\Delta \Theta_{z} \\
& \Delta \theta_{2 y}=\Delta \beta_{2}-\Delta \Theta_{y} \\
& \Delta \theta_{2 z}=\Delta \gamma_{2}-\Delta \Theta_{z} \\
& \Delta e=L_{i+1}-L_{i} \\
& \Delta \theta_{x}=\Delta \alpha_{2}-\Delta \alpha_{1}
\end{aligned}
$$

The member length $L_{\mathrm{i}}$ is calculated by

$$
L_{i}=\sqrt{\left(X_{E}^{i}\right)^{2}+\left(Y_{E}^{i}\right)^{2}+\left(Z_{E}^{i}\right)^{2}}
$$

It should be noted that the rigid body rotation increment is assumed to be finite and a rotation matrix is used to update the orientation of element local frame

$$
\begin{aligned}
& { }_{r} T= \\
& {\left[\begin{array}{ccc}
\cos \Delta \Theta_{n} & \frac{-\cos \Delta \Theta_{n} \sin \Delta \Theta_{\mathrm{y}} \cos \Delta \Theta_{\mathrm{x}}-\sin \Delta \Theta_{\mathrm{z}} \sin \Delta \Theta_{\mathrm{x}}}{\cos \Delta \Theta_{\mathrm{y}}} & \frac{\cos \Delta \Theta_{n} \sin \Delta \Theta_{\mathrm{y}} \sin \Delta \Theta_{\mathrm{x}}-\sin \Delta \Theta_{\mathrm{z}} \cos \Delta \Theta_{\mathrm{x}}}{\cos \Delta \Theta_{\mathrm{y}}} \\
\sin \Delta \Theta_{\mathrm{y}} & \cos \Delta \Theta_{\mathrm{y}} \cos \Delta \Theta_{\mathrm{x}} & -\cos \Delta \Theta_{\mathrm{y}} \sin \Delta \Theta_{\mathrm{x}} \\
\sin \Delta \Theta_{\mathrm{z}} & \frac{-\sin \Delta \Theta_{\mathrm{y}} \sin \Delta \Theta_{\mathrm{z}} \cos \Delta \Theta_{\mathrm{x}}+\sin \Delta \Theta_{\mathrm{z}} \sin \Delta \Theta_{\mathrm{x}}}{\cos \Delta \Theta_{\mathrm{y}}} & \frac{\sin \Delta \Theta_{\mathrm{y}} \sin \Delta \Theta_{\mathrm{z}} \sin \Delta \Theta_{\mathrm{x}}+\cos \Delta \Theta_{n} \cos \Delta \Theta_{\mathrm{x}}}{\cos \Delta \Theta_{\mathrm{y}}}
\end{array}\right]} \\
& \text { where } \\
& \cos \Delta \Theta_{n}=\sqrt{1-\sin \Delta \Theta_{\mathrm{y}} \sin \Delta \Theta_{\mathrm{y}}-\sin \Delta \Theta_{\mathrm{z}} \sin \Delta \Theta_{\mathrm{z}}}
\end{aligned}
$$

The element orientation matrix $\mathbf{T}$ can be updated by incremental rigid body rotation in Eq. 17 such that moderately large rotation can be accounted for. 


\subsection{Small Rotations, Small Deformational Rotations}

In this situation, the rotation can be represented by the rotational vector which is additive and the total nodal displacements can be written as,

$\mathbf{v}_{g}=\left\{u_{1}, v_{1}, w_{1}, \alpha_{1}, \beta_{1}, \gamma_{1}, u_{2}, v_{2}, w_{2}, \alpha_{2}, \beta_{2}, \gamma_{2}\right\}^{T}$

It can be updated by directly addition with the displacement increments

$\mathbf{v}_{g}=\mathbf{v}_{g}^{0}+\Delta \mathbf{v}_{g}$

in which $\Delta \mathbf{v}_{g}$ is the incremental displacements obtained an incremental-iterative predictor.

The deformational rotations can be obtained directly through the total rotations eliminating the rigid body rotations. The total rigid body rotations can be cumulated by every incremental rigid body rotation through Eq. 1 to 3 or obtained directly by the total form of these equations. Then the total local deformation $\mathbf{u}_{l}$ can be obtained through total form of Eq. 10 to 15 in which the total global displacements and total rigid body rotation are used.

The component of the total element deformation is linked with the element internal forces in secant relationship in beam-column element and can be listed as:

$\mathbf{u}_{l}=\left\{\theta_{1 y}, \theta_{1 z}, \theta_{2 y}, \theta_{2 z}, e, \theta_{x}\right\}^{T}$

\section{PEP ELEMENT FORMULATION REVIEWS}

The PEP element proposed by Chan and Zhou [9] is derived in co-rotational formulation where only basic deformations and the corresponding basic forces are involved. Besides the compatibility conditions at the two end nodes, two additional constraints of equilibrium at the mid-span of an element are considered for the evaluation of the displacement function which is a fifth-order polynomial written as follows

$v_{n}=\left(N_{1 n} N_{2 n}\right)\left(L \theta_{1 n} L \theta_{2 n}\right)^{T}$

Where $\mathrm{n}=y, \mathrm{z}$ is two principle direction of cross section, $\theta_{1 \mathrm{n}}$ and $\theta_{2 \mathrm{n}}=$ nodal rotations at two ends, $N_{1 \mathrm{n}}$ and $N_{2 \mathrm{n}}$ are shape functions which are given by

$$
\begin{aligned}
& N_{1 n}=A_{n} / H_{1 n}+B_{n} / H_{2 n} \\
& N_{2 n}=A_{n} / H_{1 n}-B_{n} / H_{2 n}
\end{aligned}
$$

And

$$
A_{n}=-20 \frac{x}{L}+\left(80-q_{n}\right)\left(\frac{x}{L}\right)^{3}+4 q_{n}\left(\frac{x}{L}\right)^{5}
$$




$$
\begin{aligned}
& B_{n}=6-\left(24-\frac{q_{n}}{2}\right)\left(\frac{x}{L}\right)^{2}-2 q_{n}\left(\frac{x}{L}\right)^{4} \\
& q_{n}=P L^{2} / E I_{n} \\
& H_{1 n}=80+q_{n} \\
& H_{2 n}=48+q_{n}
\end{aligned}
$$

The secant stiffness relationship can be formulated by the principle of stationary potential energy and is given as follows:

$$
\begin{aligned}
& M_{1 n}=\frac{E I_{n}}{L}\left[\left(c_{1 n}+c_{2 n}\right) \theta_{1 n}+\left(c_{1 n}-c_{2 n}\right) \theta_{2 n}\right] \\
& M_{2 n}=\frac{E I_{n}}{L}\left[\left(c_{1 n}-c_{2 n}\right) \theta_{1 n}+\left(c_{1 n}+c_{2 n}\right) \theta_{2 n}\right] \\
& P=E A\left[\frac{e}{L}+\sum_{n=x, y} b_{1 n}\left(\theta_{1 n}+\theta_{2 n}\right)^{2}+b_{2 n}\left(\theta_{1 n}-\theta_{2 n}\right)^{2}\right] \\
& M_{x}=\frac{G J+\operatorname{Pr}^{2}}{L} \theta_{x}
\end{aligned}
$$

In which $e=$ elongation of the element; $\mathbf{F}_{l}=\left\{M_{1 \mathrm{y}}, M_{2 \mathrm{y}}, M_{1 \mathrm{z}}, M_{2 \mathrm{z}}, P, M_{\mathrm{x}}\right\}^{\mathrm{T}}$ is local internal force vector; and $E=$ elasticity modulus; $I_{n}=$ moment of inertia; $A=$ section area; $L=$ length of the element; And $c_{1 \mathrm{n}}, c_{2 \mathrm{n}}$ and $b_{1 \mathrm{n}}, b_{2 \mathrm{n}}$ are axial force relating coefficients and can be given by

$$
\begin{aligned}
& c_{1 n}=\frac{3(80)^{2}+10(80) q_{n}+\frac{61}{7} q_{n}^{2}+\frac{23}{1260} q_{n}^{3}}{H_{1 n}^{2}} \\
& c_{2 n}=\frac{(48)^{2}+6(48) q_{n}+\frac{29}{5} q_{n}^{2}+\frac{11}{420} q_{n}^{3}}{H_{2 n}^{2}} \\
& b_{1 n}=\frac{2(80)^{2}+\frac{26}{7}(80) q_{n}+\frac{46}{21} q_{n}^{2}+\frac{23}{2520} q_{n}^{3}}{H_{1 n}^{3}} \\
& b_{2 n}=\frac{2(48)^{2}+\frac{14}{5}(48) q_{n}+\frac{66}{35} q_{n}^{2}+\frac{11}{840} q_{n}^{3}}{H_{2 n}^{3}}
\end{aligned}
$$

It should be noted that this secant stiffness relationship can be considered as the exact solution of PEP element without rigid body rotation under the condition of small deformation. 
The tangent stiffness matrix can be obtained by taking a variation of the secant stiffness with respect to the displacement degrees of freedom and the axial force due to which the second-order effect is considered. And the final expression of the incremental stiffness relationship can be written as:

${ }^{t} k_{i j}=\frac{\partial F_{l i}^{T}}{\partial u_{l j}}+\frac{\partial F_{l i}^{T}}{\partial q} \frac{\partial q}{\partial u_{l j}}$

In which $\mathrm{F}_{l i}$ and $\mathrm{u}_{l i}$ are the six components of conjugate pair of local internal forces $\mathbf{F}_{l}$ and local deformation $\mathbf{u}_{l}$. The incremental stiffness can finally be determined by the tangent stiffness matrix as,

$$
\left[\begin{array}{c}
\Delta M_{1 y} \\
\Delta M_{2 y} \\
\Delta M_{1 z} \\
\Delta M_{2 z} \\
\Delta P \\
\Delta M_{x}
\end{array}\right]=\frac{E I}{L}\left[\begin{array}{cccccc}
\zeta_{y}\left(c_{1 y}+c_{2 y}\right)+\frac{G_{1 y}^{2}}{H} & \zeta_{y}\left(c_{1 y}-c_{2 y}\right)+\frac{G_{1 y} G_{2 y}}{H} & \frac{G_{1 y} G_{1 z}}{H} & \frac{G_{1 y} G_{2 z}}{H} & 0 & \frac{G_{1 y}}{L H} \\
& \zeta_{y}\left(c_{1 y}-c_{2 y}\right)+\frac{G_{2 y}^{2} G_{2 y}}{H} & \frac{G_{2 y} G_{1 z}}{H} & \frac{G_{2 y} G_{2 z}}{H} & 0 & \frac{G_{2 y}}{L H} \\
& & \zeta_{z}\left(c_{1 z}+c_{2 z}\right)+\frac{G_{1 z}^{2}}{H} & \zeta_{z}\left(c_{1 z}-c_{2 z}\right)+\frac{G_{1 z} G_{2 z}}{H} & 0 & \frac{G_{1 z}}{L H} \\
& & & \zeta_{z}\left(c_{1 z}+c_{2 z}\right)+\frac{G_{2 z}^{3}}{H} & 0 & \frac{G_{2 z}}{L H} \\
& \text { symmetrical } & & \eta & 0 \\
& & & \frac{1}{L H}
\end{array}\right]
$$

in which $G_{1 n}, G_{2 n}$ and $H$ are parameters given by

$G_{1 n}=2 b_{1 n}\left(\theta_{1 n}+\theta_{2 n}\right)+2 b_{2 n}\left(\theta_{1 n}-\theta_{2 n}\right)$

$G_{2 n}=2 b_{1 n}\left(\theta_{1 n}+\theta_{2 n}\right)-2 b_{2 n}\left(\theta_{1 n}-\theta_{2 n}\right)$

$H=\frac{1}{\lambda^{2}}-\sum_{n=y, z} \frac{b_{1 n}^{\prime}\left(\theta_{1 n}+\theta_{2 n}\right)^{2}+b_{2 n}^{\prime}\left(\theta_{1 n}-\theta_{2 n}\right)^{2}}{\zeta_{n}}$

$\eta=\frac{G J+\operatorname{Pr}^{2}}{E I}$

where

$$
\begin{aligned}
& \lambda=\sqrt{\frac{A L^{2}}{I}} \\
& \zeta_{n}=\frac{I_{n}}{I}
\end{aligned}
$$




\section{FORCE RECOVERY PROCEDURE}

When the corrector or force recovery procedure is exercised in the incremental iterative analysis, the element deformation ${ }^{0}\left[\mathrm{u}_{l}\right]$ in last configuration and the present deformation increments ${ }^{0}\left[\Delta \mathrm{u}_{l}\right]$ are extracted from the incremental displacement computed by the predictor equations through Eq. 10 to 15 . The task of the force recovery procedure is to recover the element internal forces ${ }^{0}\left[\mathrm{~F}_{l}\right]$ $+\left[\Delta \mathrm{F}_{l}\right]$ at the current configuration. There are mainly three methods to complete this task.

\subsection{Total Secant Iteration Method}

Like other beam-column elements, the PEP element has explicit secant stiffness relationship. So in the corrector phase the element internal forces can be calculated directly by the secant stiffness in Eq. 30 to 32 with the present element deformation $\left[\mathrm{u}_{l}\right]$ cumulated from the element deformation ${ }^{0}\left[\mathrm{u}_{l}\right]$ in last configuration and the present deformation increments ${ }^{0}\left[\Delta \mathrm{u}_{l}\right]$. It should be noted that $c_{1 \mathrm{n}}, c_{2 \mathrm{n}}$ and $b_{1 \mathrm{n}}, b_{2 \mathrm{n}}$ are axial force relating coefficients and is calculated based on the axial force of last iteration. Iteration must be used for more accurate results.

\subsection{Force Incremental Method}

The prevalent approach to calculate the element recovery forces at each incremental step in finite element method is cumulated from the force increments. In co-rotational method the displacement increments $\Delta \mathrm{u}$ can be decomposed into two parts: the rigid body displacements $\Delta \mathrm{u}_{r}$ and the natural deformations $\Delta \mathrm{u}_{l}$. The effect of the rigid body displacements $\Delta \mathrm{u}_{r}$ is to rotate the initial nodal forces from the last configuration to the present configuration without generation of internal forces. So the element force increments can be calculated by the natural deformations as,

$$
\Delta f_{l}=k_{n}^{e} \Delta u_{l}
$$

in which $\boldsymbol{k}_{\mathrm{n}}^{\mathrm{e}}$ is the element natural stiffness matrix. The accuracy of force increments is only used in the first order of the displacement increments so every load cycle step should be small enough to obtain a sufficiently accurate solution. The internal forces of the element can be updated by firstly adding the incremental internal nodal forces as,

${ }^{i+1} F_{e}={ }^{i} F_{e}+\Delta f_{e}$

then the nodal forces from the last configuration are rotated to the present configuration through member orientation matrix.

Chan [2] proposed an incremental secant formulation based on UL formulation and Cubic element in which the same incremental stiffness is used as both the predictor and corrector. So actually it is same with this force incremental method. It is also pointed out that this incremental method is better than total secant formulation in dealing with non-vectorial rotations and has merits for use in the genuinely large rotation case.

\subsection{Incremental Secant Iteration Method}

The relationship between the element internal forces with the element deformations of last configuration in Eqs. 30-33 can be written in a simplified form as

$F_{l}=\varphi\left(F_{l}, u_{l}\right)$ 
Given the deformation increments ${ }^{0}\left[\Delta \mathrm{u}_{l}\right]$, the element internal forces cannot be calculated directly as the above functions are a set of implicit nonlinear functions of element internal forces $\mathbf{F}_{l}$. To solve this problem, the inverse form of the above equations can be written as

$$
u_{l}=\psi\left(F_{l}, u_{l}\right)
$$

These nonlinear equations can be solved readily by the Newton-Raphson procedure used in the analysis of nonlinear systems and the iterative procedure can be written as

$$
\begin{aligned}
& { }^{i}\left[\Delta F_{l}\right]={ }^{i}\left[\frac{\partial u_{l}}{\partial F_{l}}\right]^{-1}\left[\Delta u_{l}\right]={ }^{i}\left[\frac{\partial F_{l}}{\partial u_{l}}\right]{ }^{i}\left[\Delta u_{l}\right]={ }^{i}[k]^{i}\left[\Delta u_{l}\right] \\
& { }^{i+1}\left[F_{l}\right]={ }^{i}\left[F_{l}\right]+{ }^{i}\left[\Delta F_{l}\right] \\
& { }^{i+1}\left[\Delta u_{l}\right]={ }^{0}\left[u_{l}\right]+{ }^{0}\left[\Delta u_{l}\right]-{ }^{i}\left[u_{l}\right]
\end{aligned}
$$

The iteration process of Eqs. 50-52 continues until the unbalanced deformation ${ }^{\mathrm{i}}\left[\Delta \mathrm{u}_{l}\right]$ is sufficiently small. In this iteration the total deformation ${ }^{\mathrm{i}}\left[\mathrm{u}_{l}\right]$ can be easily calculated by the reverse form of Eqs. 30-33. And it should be noted that the tangent matrix in Eq. 50 is simply the element tangent stiffness matrix and the incremental properties can be found from equation Eq. 51 and Eq. 52 .

\subsection{Comparison of Different Methods}

Strictly speaking, the aforementioned force incremental method will introduce errors inevitable cause the element tangent stiffness instead of accurate secant stiffness is used to calculate recovery forces. As illustrated by Figure 2, the exact element forces that should be obtained in force recovery phase is point A but with force incremental method we can only get point B. According to our experience in numerical analysis, the stability and convergent speed of the total secant iteration method are better than that of the pure incremental iteration in the case of moderately large load step size. This is reasonable as the accuracy of force increments can only be ensured with sufficiently small step size while the secant stiffness is accurate so long as the deformation is not large and the accuracy becomes unrelated to the step size.

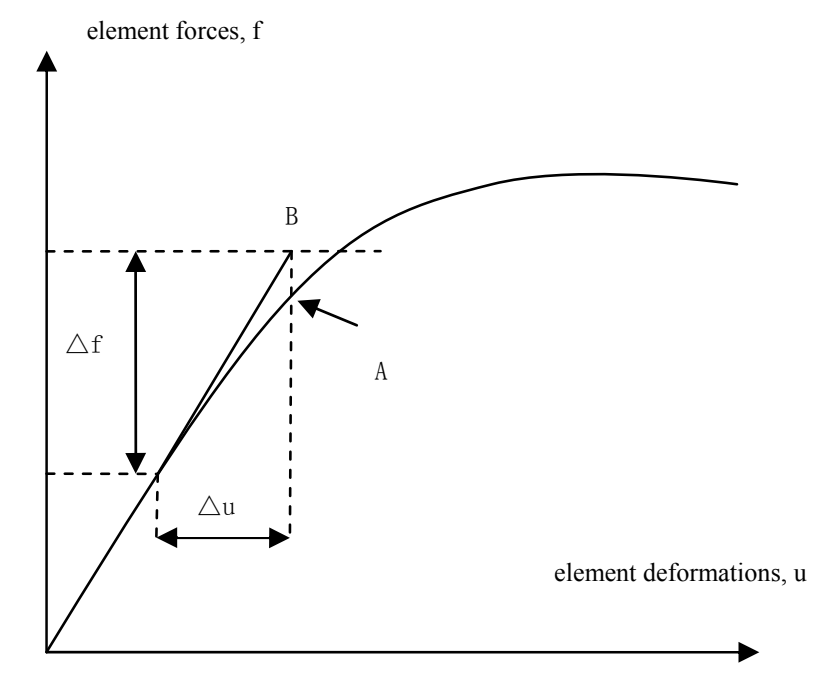

Figure 2. Illustration of Force Recovery Procedure 
As far as material nonlinearity such as semi-rigid connection or plastic hinge analysis is concerned, the incremental force recovery method is more suitable as the material nonlinearity is incremental in nature. If plasticity is involved, the cross-section strength or yield state is determined by the de-composition of axial force and moments. A procedure is required to be adopted for bringing back a force point lying outside the full yield surface to this yield surface as the yield function cannot be violated with any point outside the surface is a non-defined state for the element. With ${ }^{0}\left[\mathrm{~F}_{l}\right]$ and $\left[\Delta \mathrm{F}_{l}\right]$, it can be easily concluded if the next force point is outside the full yield surface and the appropriate increment step can be adjusted to avoid a non-defined state. The total secant iteration method has better accuracy and faster convergent rate than force incremental method with moderately large step size but have defect in coping with plasticity as the force recovery procedure is not incremental. With the proposed incremental secant iteration method, the correct recovery forces are obtained and incremental manner is preserved which is crucial in the prediction of formation of plastic hinges in the solution process.

\section{NUMERICAL EXAMPLES}

\subsection{A Simple Cantilever}

A simple cantilever shown in Figure 3 is analyzed to illustrate the different convergence properties of the three force recovery methods. A horizontal compressive force is applied to the tip of the cantilever with a transverse disturbance load of $0.1 \% \mathrm{P}$ to simulate imperfections. Only one element is used for the analysis of the cantilever.

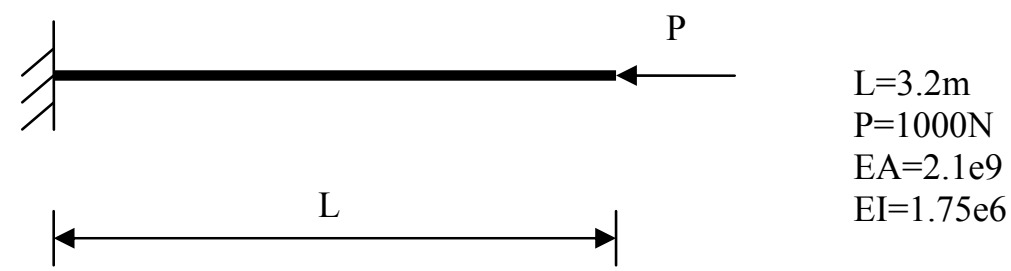

Figure 3. A Simple Cantilever

Figures 4 and 5 show the load deflection paths obtained by the three force recovery methods in conjunction with Newton-Raphson load control method and the load increments are 1 and 0.2 respectively. It can be found that the load deflection curves obtained by the total secant iteration and the incremental secant iteration methods are exactly same while the force incremental method haves apparent drift with that of the other two methods, especially when the load increments are large. And the drift occurs at mid segment of the curve where the stiffness of the structure is very small and a small load increment will lead to a large displacements. This is because the accuracy of force increments obtained by force incremental iteration method is only in the first order of the displacement increments so it is not load size but the displacements incremental size determines the accuracy of the solution. It is recommended that single displacement control or arc length control scheme be used with the force incremental iteration method. This can be confirmed in Figure 6 where very small arc length constraint needs to be specified for every step and nearly the same curves are obtained by three different recovery methods. 


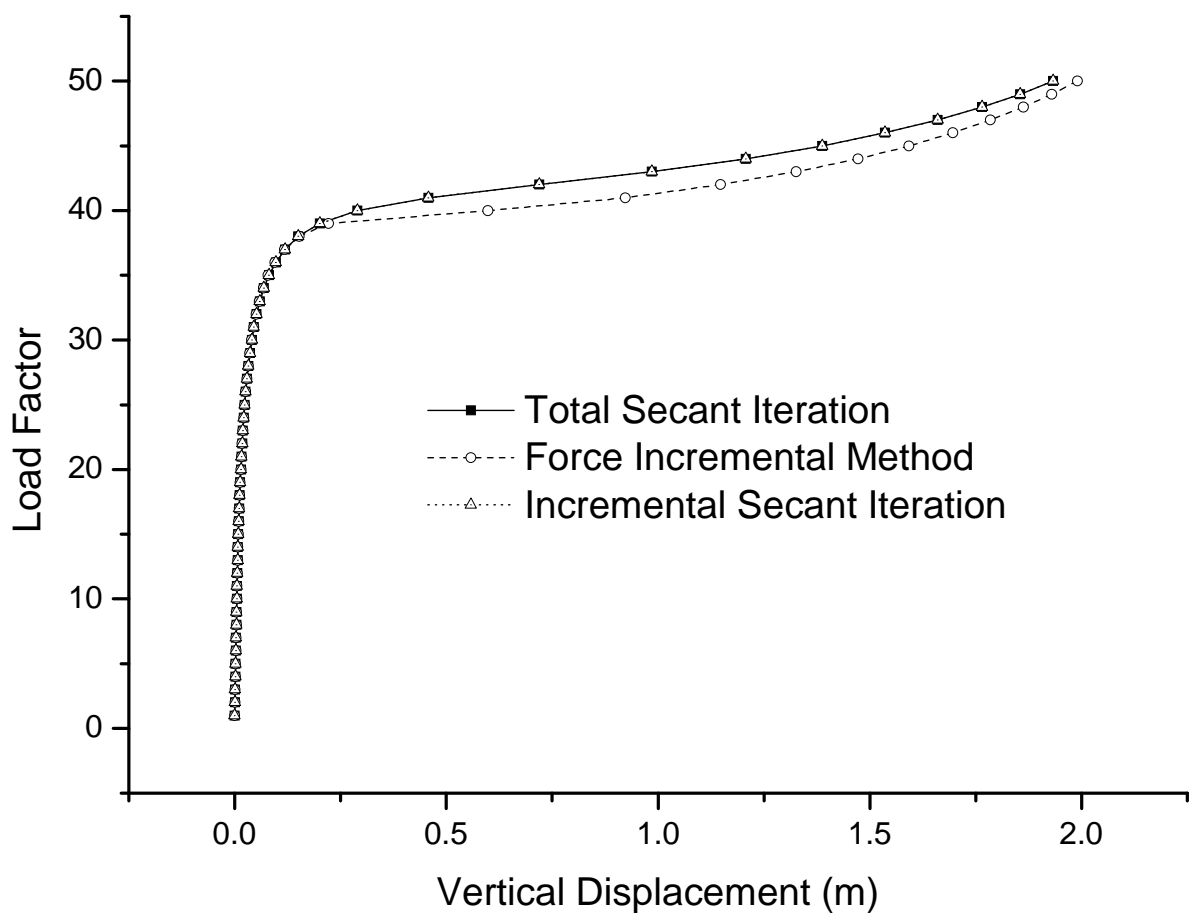

Figure 4. Load Displacement Path with Every Load Increment of 1

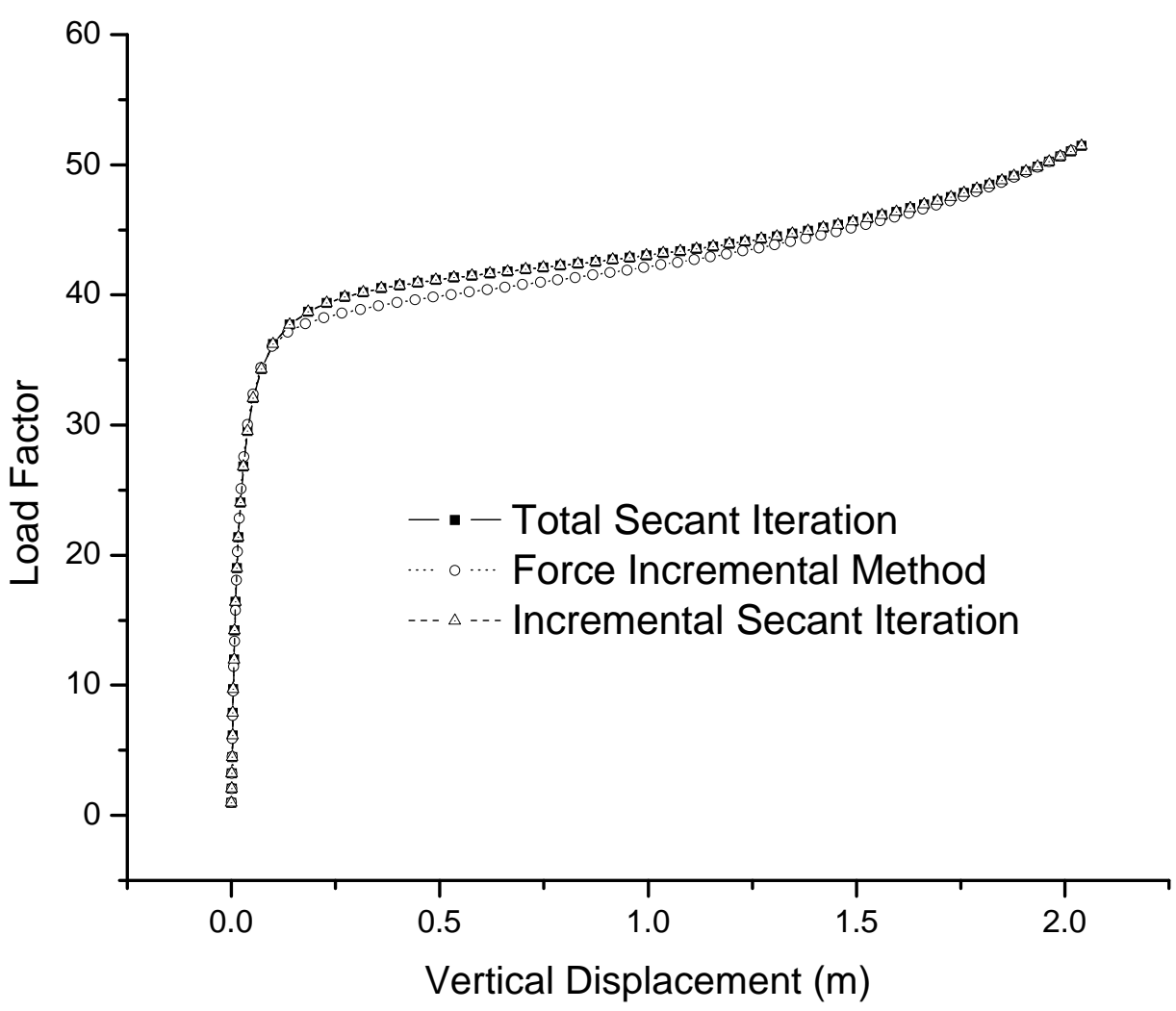

Figure 5. Load Displacement Path with Every Load Increment of 0.2 


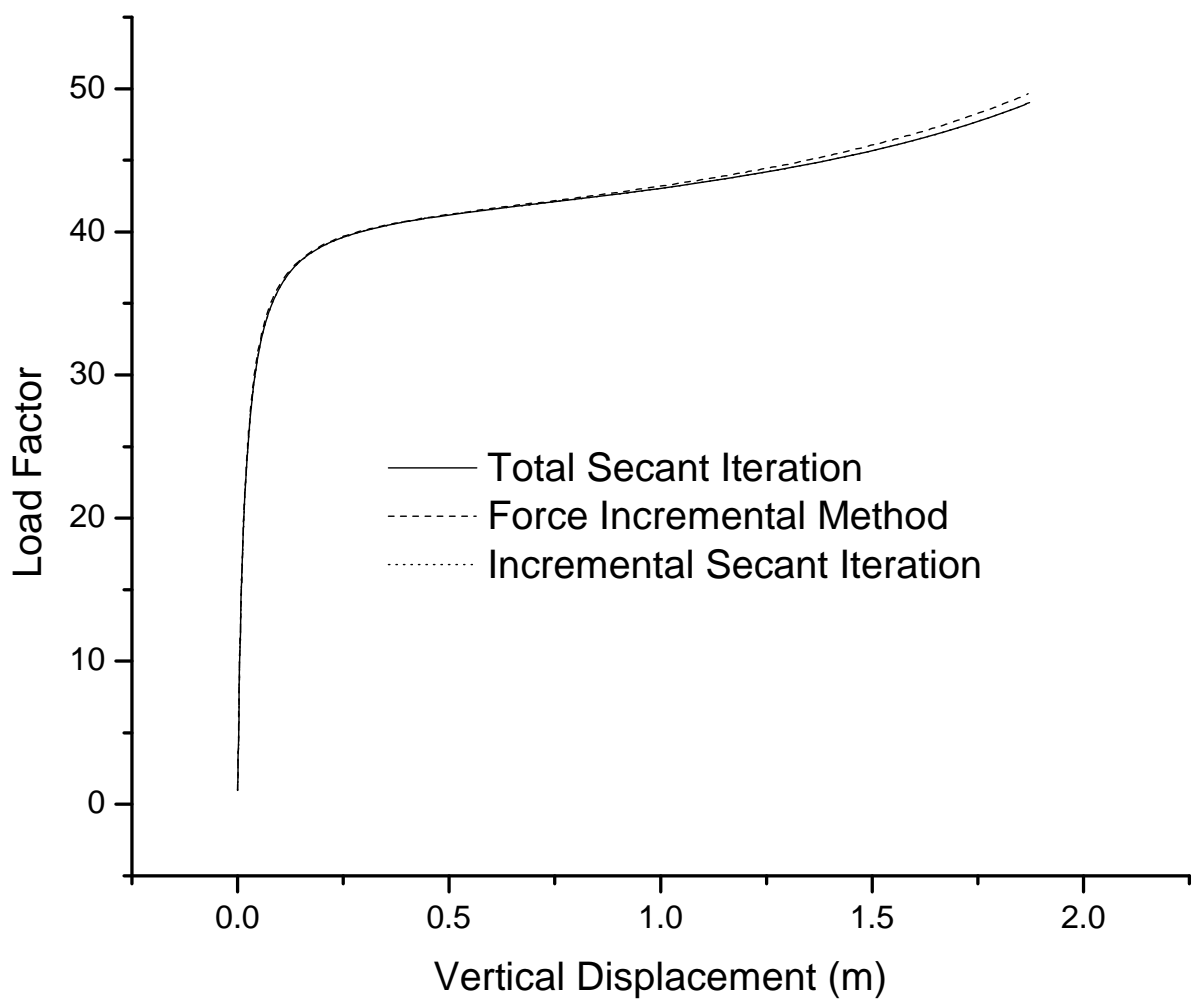

Figure 6. Load Displacement Path with Arc Length Method

\subsection{William Toggle}

Figure 7 shows the well-known William toggle frame which has been extensively used to investigate the snap-through buckling behavior. The Young's modulus of elasticity is $1.0 \times 103$ $\mathrm{N} / \mathrm{cm}^{2}$. The axial rigidity (EA) is $18550 \mathrm{~N}$ and the flexural rigidity (EI) is $9270 \mathrm{Ncm}^{2}$. Only one element is used to model each inclined member and semi-rigid springs with flexural rigidity of $1 / 10$ EI is attached to the two ends of elements. From Figure 8 it can be seen that almost same load deflection paths can be obtained by the three force recovery methods in conjunction with arc length method when the displacement is not large and there is significant differences between the force incremental method and the other two secant related methods at the latter portion of the curves where the deformations of members are large.

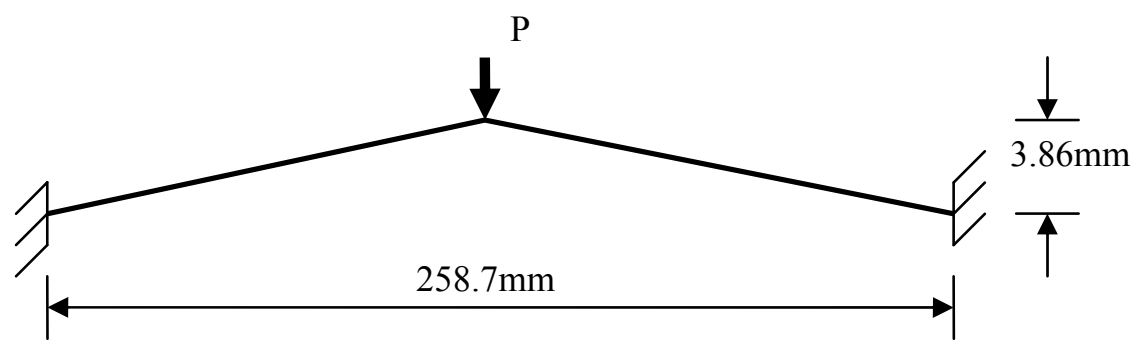

Figure 7. William Toggle 


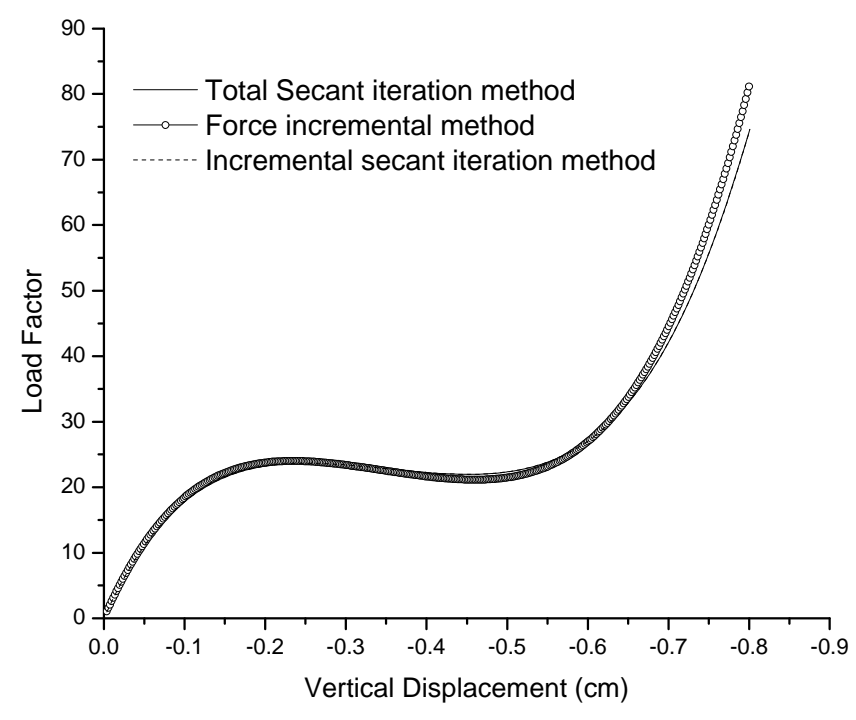

Figure 8. Load Displacement Path of Toggle Frame

\subsection{Member 3-Dimensional Space Frame}

An example of a 90-member hexagonal shallow dome with its dimensions and properties shown in Figure 9 is under a set of downward forces of $100 \mathrm{~N}$ each acting on the non-supporting nodes. Only one single PEP element is used to model a member and member initial imperfection of magnitude equal to $1 / 1000^{\text {th }}$ of member length is assumed for every curved PEP element. Members of a shallow dome are typically under high axial forces because of the arch action and such an arrangement for large nodal displacement and rotation is useful to validate the performance of the proposed force recovery method.

Figures 10 and 11 show the load displacement path of vertical and lateral deflection respectively. Almost the same curves are obtained by the three force recovery methods with minimum residual displacement method in used with small step size .
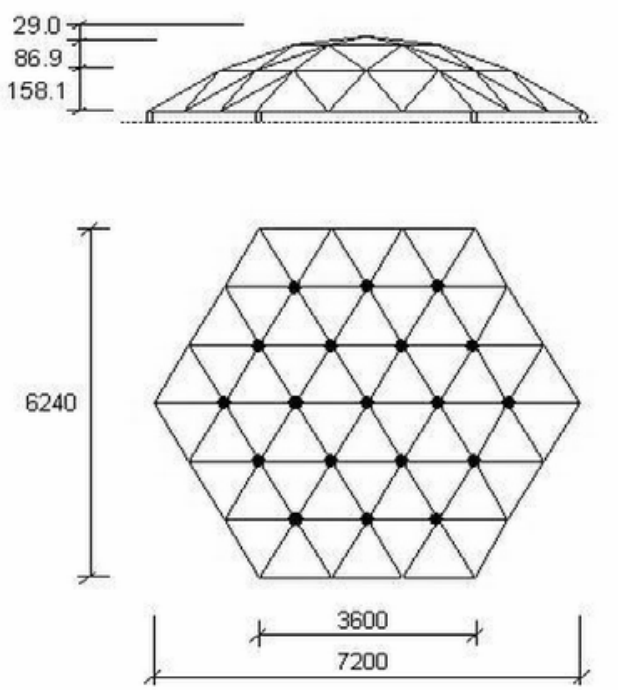

$$
\begin{aligned}
& E=1.95 e 5 \mathrm{~N} / \mathrm{mm}^{2} \\
& G=0.80 e 5 \mathrm{~N} / \mathrm{mm}^{2} \\
& A=142.3 \mathrm{~mm}^{2}
\end{aligned}
$$

$$
\text { ly }=1.44 \mathrm{e}^{4} \mathrm{~mm}^{4}
$$$$
\mathrm{Iz}=1.44 \mathrm{e}^{4} \mathrm{~mm}^{4}
$$$$
\mathrm{J}=2.89 \mathrm{e}^{4} \mathrm{~mm}^{4}
$$

Unit: $\mathrm{mm}$

- Loaded node

Figure 9. Hexagonal Shallow frame 


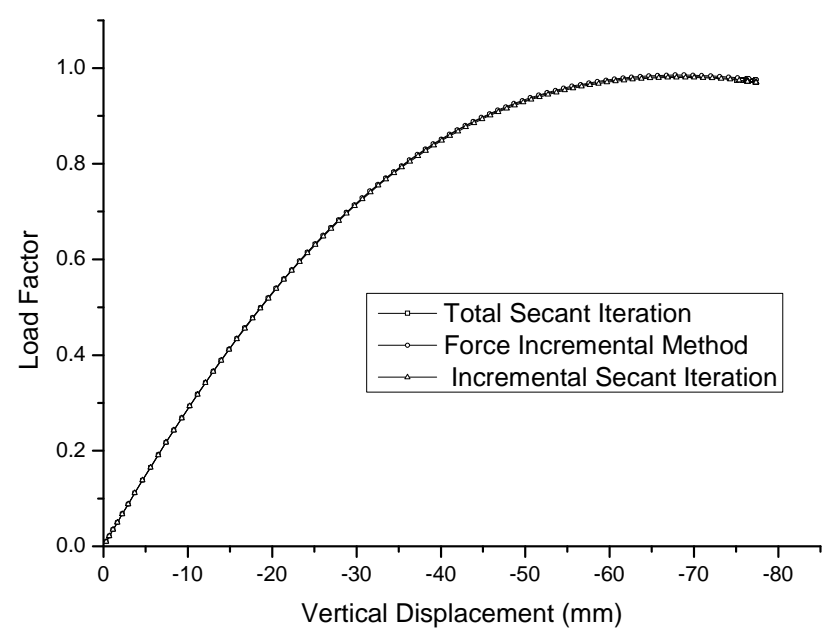

Figure 10. Load-Vertical Displacement Path of Hexagonal Shallow Frame

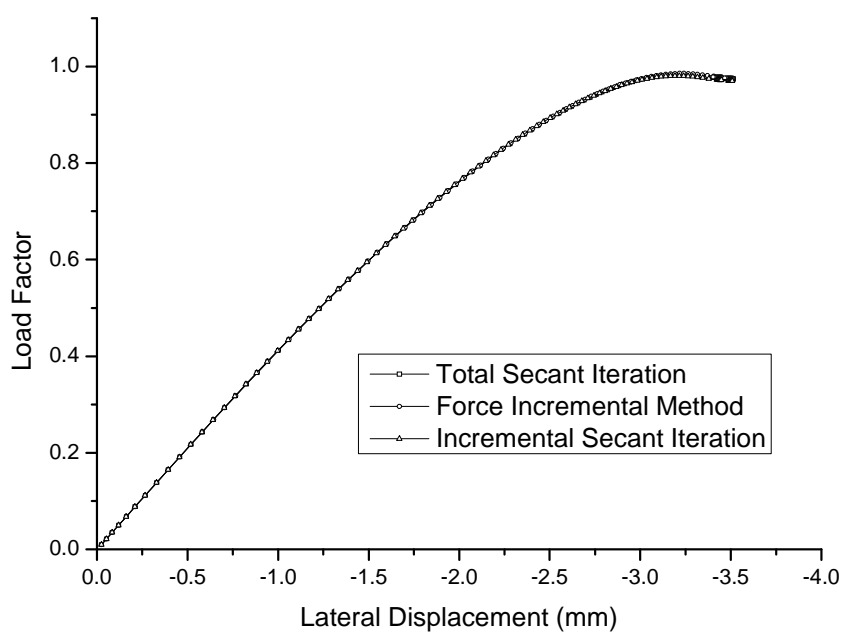

Figure 11. Load-Lateral Displacement Path of Hexagonal Shallow Frame

\section{CONCLUSION}

In this paper the recovery procedure of the element forces is investigated and three force recovery methods, namely as the total secant iteration, force incremental and the incremental secant iteration force recovery procedures are detailed and numerical results by these three force recovery methods are compared. It is found that the three force recovery methods can get almost the same result if we make the increments sufficiently small. And the total secant iteration and the incremental secant iteration can always get the same result irrespective of the step size used. The force incremental method has a considerable drift with that of the other two methods when the step size is large and the difference is minimized when small load/displacement step size is used. The incremental characteristic of force recovery procedure is important to forecast the formation of a plastic hinge and thus engineer should be aware of the limitation of various formulations. The proposed incremental secant iteration method can compute the internal forces in an explicit incremental manner and has the same convergence characteristics identical to the total secant iteration. The application of the proposed method in semi-rigid connection and plastic hinge analysis will be illustrated after further investigation. 


\section{REFERENCES}

[1] Yang, Y.B., Lin, S.P. and Leu, L.J., "Solution Strategy and Rigid Element for Nonlinear Analysis of Elastically Structures Based on Updated Lagrangian Formulation", Engineering Structures, 2007, Vol. 29, pp. 1189-1200.

[2] Chan, S.L., "Large Deflection Kinematic Formulations for Three-Dimensional Framed Structures", Computer Methods in Applied Mechanics and Engineering, 1992, Vol. 95, pp. 17-36.

[3] Oran, C., "Tangent Stiffness in Space Frames", 1973, Vol. 99, pp. 987-1001.

[4] Izzuddin, B.A. and Elnashai, A.S., "Eulerian Formulation for Large-Displacement Analysis of Space Frames", Journal of Engineering Mechanics, 1993, Vol. 119, pp. 549-569.

[5] Felippa, C.A. and Haugen, B., "A Unified Formulation of Small-Strain Co-Rotational Finite Elements: I. Theory", Computer Methods in Applied Mechanics and Engineering, 2005, Vol. 194, pp. 2285-335.

[6] Chan, S.L. and Kitipornchai, S., "Geometric Nonlinear Analysis of Asymmetric Thin-Walled Beam-Columns", 1987, Vol. 9, pp. 243-254.

[7] Papadrakakis, M., "Post-Buckling Analysis of Spatial Structures by Vector Iteration Methods", Computers and Structures, 1981, Vol. 14, pp. 393-403.

[8] Chin, C.K., Al-Bermani, F.G.A. and Kitipornchai, S., "Non-Linear Analysis of Thin-Walled Structures Using Plate Elements", International Journal for Numerical Methods in Engineering, 1994, Vol. 37, pp. 1697-1711.

[9] Chan, S.L. and Zhou, Z.H., "Pointwise Equilibrating Polynomial Element for Nonlinear Analysis of Frames", Journal of Structural Engineering, 1994, Vol. 120, pp. 1703-1717. 